\title{
Presa in carico infermieristica nella Malattia Renale Cronica
}

\author{
Maria Pia Zito ${ }^{1}$, Roberta Toschi ${ }^{2}$ \\ ${ }^{1}$ SIAN, Società Infermieri Area Nefrologica, Bologna - Italy \\ ${ }^{2}$ Consigliere Ordine delle Professioni Infermieristiche di Bologna - Italy
}

\begin{abstract}
Nursing Management in Chronic Kidney Disease
In 2017, thanks to an initiative of IPASVI College (the Order of Nursing Professionals of the province of Bologna) in response to 2014 legislations referring to the Guidelines about Chronic Kidney Disease (CKD), nephrology professionals and members of EDTNA/ERCA Italian Branch Association (which in 2018 became Società Infermieri Area Nefrologica, SIAN) in 2021 were requested to contribute to define the role and skills performed by nurses on a daily basis.

The management of the CKD patient has been structured into 7 phases: the model for each phase takes into consideration the professionals involved, the care settings and the tools used.

To support this approach, we decided to introduce two elements which broaden the clinical and care approach to the patient and at the same time highlight the contribution of nursing professionals, with a referring workflow for the CKD patient, the nurses' role and skills required throughout the patient's journey.

Furthermore, to ensure the best continuity of care, we believe that it is necessary to apply a logical model based on the principles of early recognition of health and welfare needs; guarantee the most appropriate healthcare response; ensure early care; standardize the procedures of professionals to improve the quality of life of the patient and manage the social and economic impact.
\end{abstract}

Keywords: CKD, CKD patients management, Nursing skills

\section{Introduzione}

Questo documento nasce come quaderno della formazione dell'Ordine delle Professioni Infermieristiche (OPI) di Bologna, da un Gruppo Infermieristico impegnato nelle varie realtà cliniche e organizzative nefrologiche provinciali, costituito nel 2017 per precisa volontà del Consiglio Direttivo dell'allora Collegio Ipasvi, provincia di Bologna, in risposta al neo emanato documento ministeriale riguardante le Linee Guida sul percorso della Malattia Renale Cronica (MRC). L'obiettivo era definire il ruolo e le competenze dell'Infermiere nell'assistenza al paziente con Malattia Renale Cronica, come Integrazione alle Linee Guida Ministeriali sul Percorso della Malattia Renale Cronica 2014.

Received: August 3, 2021

Accepted: August 5, 2021

Published online: September 22, 2021

Indirizzo per la corrispondenza:

Maria Pia Zito

Tesoriera SIAN

Via Montanara 8/3

50055 Castenaso (BO) - Italy

mariapiazito1@gmail.com
Le Linee Guida ministeriali, se, da un lato, superano il percorso classico paziente/malattia renale cronica/nefrologo, introducendo nuovi percorsi diagnostici e terapeutici con interventi coordinati e integrati di professionisti diversi, non esplicitano in maniera chiara il ruolo dell'Infermiere e le peculiarità educative e assistenziali. Nel Documento si mette in risalto il ruolo del Personale Sanitario, nel tentativo di comprendere tutti i professionisti coinvolti, ma, a nostro parere, limitando, relegando e non chiarendo il sostanziale apporto infermieristico.

È stato chiesto, pertanto, ai Professionisti impegnati nelle varie realtà cliniche e organizzative nefrologiche e ad alcuni componenti dell'Associazione EDTNA/ERCA Filiale Italiana e, dal 2018, Società Infermieri Area Nefrologica (SIAN) di dare il loro contributo affinché emergessero il ruolo e le competenze portati avanti quotidianamente dagli Infermieri. All'interno del gruppo è stata prevista anche la presenza di Professionisti dell'area Cure Primarie, poiché il Percorso, a nostro giudizio, deve includere elementi di prevenzione primaria e secondaria. Inoltre, si è volutamente adottata un'ottica diversa, dando, così, una lettura assistenziale del profilo del paziente (Assistenza al Paziente con MRC) e non della malattia (Percorso della MRC), così come proposto nelle Linee Guida di riferimento (1), più specificatamente di natura clinica. 
Con questo Documento ci auguriamo di fornire una lettura critica delle Linee Guida, fornendo, al contempo, integrazione e completamento di alto profilo Assistenziale e auspicando possa essere inserito a pieno titolo all'interno dei documenti che recepiranno le Linee Guida ministeriali nella regione Emilia Romagna.

\section{Ipotesi di percorso}

Le Linee Guida "Documento di indirizzo per la Malattia Renale Cronica", emanate dal Ministero della Salute nel 2014, rappresentano senza dubbio un'evoluzione nella presa in carico del paziente nefropatico.

Il paziente nefropatico rappresenta "il tipico esempio di cronicità della malattia", il cui intervento richiede un approccio di squadra e una strutturazione dei processi di cura. Nel corso del suo cammino verso la cronicità, il paziente va incontro a esigenze cliniche diverse, strutturate su differenti livelli che sono comunque parte di un unico processo di cura.

La prevenzione e l'identificazione dei soggetti a rischio assumono un'importanza centrale nella gestione della patologia, e lo stesso vale per l'educazione terapeutica volta a migliorare l'adesione del paziente alle cure e alle indicazioni clinico-assistenziali.

Fondamentale il coinvolgimento di diverse figure professionali, team multidisciplinare (Nefrologo, Infermiere, Infermiere Case Manager, Dietista, Psicologo, Assistente Sociale, Chirurgo vascolare).

A sostegno della validità della scelta, si pensa, tuttavia, di introdurre due elementi che ampliano l'approccio clinico e assistenziale al paziente e che, al contempo, evidenziano I'apporto e le competenze degli Infermieri:

1. uno schema di riferimento del percorso del paziente con Malattia Cronica Renale;

2. il ruolo e le competenze degli Infermieri impegnati in tutto il percorso del paziente.

Si propone uno schema di riferimento (Fig. 1) basato sul paziente inserito in un percorso educativo, assistenziale e clinico.

Un percorso che inizia con l'informazione e la sensibilizzazione (prevenzione primaria) e che trova la sua conclusione con la terapia sostitutiva (dialisi) o con il trapianto.

Ogni professionista che compone il team multiprofessionale (Medico di Medicina Generale (MMG), Nefrologo, Infermiere, Psicologo, Dietista, altri specialisti) interviene, nelle varie fasi del percorso, con una logica di funzioni e competenze in risposta dei bisogni della persona in un'ottica educativa, clinica e assistenziale complessiva.

\section{Ruolo e competenze degli Infermieri}

Come sopra riportato, le Linee Guida Ministeriali non sono esaustive nel declinare l'importante e sostanziale ruolo
dell'Infermiere all'interno dei nuovi percorsi diagnostici e terapeutici, quindi, per garantire la migliore continuità assistenziale, riteniamo sia necessario mettere in campo un modello logico che si basi su principi fondamentali:

- riconoscere precocemente i bisogni sanitari e socioassistenziali;

- garantire la più appropriata risposta assistenziale;

- garantire una presa in carico precoce;

- uniformare le procedure dei Professionisti, al fine di migliorare la qualità della vita della persona assistita e di gestire l'impatto sociale ed economico.

\section{Fasi del Modello}

Il modello (Fig. 1) per ogni fase, prende in considerazione le figure professionali interessate, i setting assistenziali e gli strumenti utilizzati, come illustrato nel documento ministeriale pubblicato nel 2014 (2).

\section{Percorso del paziente in sette fasi}

1. Informazione e sensibilizzazione

2. Screening di patologia nefrologica e riscontro di fattori predisponenti indici iniziali di MRC

3. Diagnosi di MRC progressiva e di ESRD e avvio del paziente a un percorso strutturato di presa in carico precoce

4. Avviamento al percorso pre-dialisi

5. Presa in carico dei pazienti late referral: Insufficienza renale acuta (IRA), Lesione renale acuta, (AKI), Fasi della malattia cronica (CKD), Malattia renale allo stadio terminale (ESRD)

6. Dialisi peritoneale ed emodialisi

7. Trapianto di rene

\section{$1^{\text {a }}$ fase}

Informazione e sensibilizzazione

Attuata dall'Infermiere in tutti i contesti del territorio (scuole, eventi divulgativi pubblici, Servizi sanitari ecc.), anche in collaborazione con altri Professionisti sanitari.

- L'Infermiere partecipa attivamente alle campagne informative e di sensibilizzazione alla prevenzione delle nefropatie.

- Partecipa all'identificazione dei soggetti che presentano fattori di rischio relativi, anche con azioni rispondenti alle logiche della Medicina d'Iniziativa.

\section{$2^{\text {a }}$ fase}

Screening Patologia Nefrologica e Riscontro fattori predisponenti indici iniziali di MRC

- Fase specificatamente clinica in cui l'Infermiere può avere un ruolo collaborativo e di supporto. 


\section{Elementi di assistenza infermieristica}

\begin{tabular}{|c|c|c|c|}
\hline $\begin{array}{l}\text { Aspetti } \\
\text { tecnici }\end{array}$ & $\begin{array}{c}\text { Aspetti } \\
\text { professionali }\end{array}$ & $\begin{array}{l}\text { Aspetti } \\
\text { educativi }\end{array}$ & $\begin{array}{c}\text { Aspetti } \\
\text { organizzativi }\end{array}$ \\
\hline
\end{tabular}

\begin{tabular}{|c|c|c|c|}
\hline \multicolumn{4}{|c|}{ Suddivisione del percorso della MRC } \\
\hline \multirow[t]{3}{*}{$\begin{array}{l}\text { 1. Informazione e } \\
\text { sensibilizzazione }\end{array}$} & \multirow{2}{*}{$\begin{array}{l}\text { 2. Screening patologia } \\
\text { nefrologica e riscontro } \\
\text { indici iniziali di MRC } \\
\text { 3. Diagnosi di MRC e } \\
\text { ESRD. }\end{array}$} & $\begin{array}{l}\text { 4. Avviamento al } \\
\text { percorso pre-dialisi }\end{array}$ & $\begin{array}{l}\text { 5. Presa in carico pazienti } \\
\text { late referral: IRA, AKI, } \\
\text { CKD, ESRD }\end{array}$ \\
\hline & & & 6. Dialisi peritoneale ed \\
\hline & $\begin{array}{l}\text { Avvio paziente in } \\
\text { percorso strutturato di } \\
\text { presa in carico precoce }\end{array}$ & & 7. Trapianto di rene \\
\hline Presa in carico & \multicolumn{2}{|c|}{ Follow-up } & Complicanze \\
\hline \multicolumn{4}{|c|}{ Aspetti clinico-assistenziali } \\
\hline
\end{tabular}

Fig. 1 - Schema di riferimento.

\section{$3^{\text {a }}$ fase}

Diagnosi di MRC progressiva e di ESRD. Avvio dei soggetti individuati a un percorso strutturato

- Presa in carico precoce dei pazienti, inviati dagli Ambulatori Nefrologici agli Ambulatori Infermieristici di percorso/ cronicità, con interventi di educazione terapeutica, finalizzati all'autogestione della malattia nelle fasi iniziali al fine di rallentarne la progressione, agendo sui modelli funzionali con un approccio assistenziale olistico.

- Assessment assistenziale della persona assistita e dell'ambito familiare, al fine di cogliere l'intervento adeguato e mirato allo stadio della MRC e alle scelte terapeutiche possibili.

- Promozione e sviluppo (in collaborazione con un team strutturato e multidisciplinare) di strategie di adattamento/coping alla malattia da parte del paziente, per migliorare la propria qualità di vita.

- Nel territorio, sviluppa attività mirate alla presa in carico domiciliare e/o ambulatoriale. Effettua la sua assistenza attraverso un Counseling mirato, rafforzando le raccomandazioni e le informazioni già ottenute nei contesti specialistici in tema di Educazione Terapeutica, nell'ottica dell'Empowerment del paziente e del caregiver nella gestione della malattia. Con i Professionisti esperti, Medici, Infermieri Specialisti e Dietisti collaborano nell'attuazione dei controlli clinico-diagnostici programmati.

\section{$4^{\mathrm{a}}$ fase}

Avviamento al percorso Pre-Dialisi

Il percorso è caratterizzato da incontri/visite/colloqui programmati con l'Infermiere Specialista e con il medico Nefrologo, sulla base delle necessità cliniche della persona assistita, riguardante:

- ausilio del trattamento sostitutivo (emodialisi o dialisi peritoneale domiciliare);

- avvio all'allestimento dell'accesso vascolare per emodialisi: fistola artero-venosa (FAV) o inserimento di Catetere 
Venoso Centrale (CVC) per esaurimento del letto vascolare superficiale o posizionamento di catetere peritoneale;

- promozione all'inserimento in lista d'attesa per trapianto renale e valutazione idoneità al trapianto;

- assessment assistenziale della persona assistita e dell'ambito familiare, al fine di cogliere l'intervento adeguato e mirato allo stadio della MRC e alle scelte terapeutiche possibili.

L'aspetto educativo assume un ruolo fondamentale anche in questa fase terminale della patologia, in quanto, con il suo aggravarsi, sono necessarie informazioni sempre più accurate riguardo la malattia stessa e la sua gestione. In pre-dialisi è importante anche l'aspetto psicologico di ascolto, dove l'Infermiere svolge un ruolo di mediatore tra il medico ed eventualmente lo psicologo, il paziente, la famiglia e la malattia.

\section{$5^{\text {a }}$ fase \\ Presa in carico del paziente Late Referral (IRA/AKI/CKD ESRD)}

I pazienti in IRA/ESRD possono essere trattati nei centri specifici di dialisi o in reparti di degenza/terapie intensive.

In questa fase, l'Infermiere mette in atto l'esperienza e la capacità di prendere in carico la persona che manifesta un problema nefrologico acuto, monitorando il paziente nelle sue funzioni vitali e somministrando un trattamento terapeutico prescritto nelle modalità più appropriate al momento. Mostra capacità di gestione della relazione d'aiuto con la persona assistita e la sua famiglia, supportandola anche in certi aspetti psicologici dell'accettazione della difficoltà momentanea e/o della malattia.

Educa all'autocura la persona con problemi nefrologici e sviluppa e realizza interventi di educazione terapeutica continua.

\section{6 fase}

\section{Dialisi peritoneale ed emodialisi}

L'Infermiere Specialista in possesso di abilitazione al trattamento dialitico:

- appartiene a un gruppo multidisciplinare;

- pianifica e attua il trattamento individuato/prescritto dal personale medico attraverso le conoscenze acquisite e le applica correttamente alle apparecchiature dialitiche e in risposta ai bisogni specifici del paziente dializzato;

- gestisce gli accessi vascolari allestiti per il trattamento sostitutivo;

- applica le prescrizioni terapeutiche, valutando costantemente le condizioni del paziente, riconoscendo precocemente le problematiche e risolvendole con competenza professionale;

- pianifica e attua interventi organizzativi miranti a soddisfare i bisogni del malato cronico in trattamento dialitico ospedaliero e domiciliare;
- produce protocolli e procedure atte a migliorare l'assistenza infermieristica e settoriale;

- pianifica un percorso di training, educando la persona assistita e/o il caregiver/partner alla gestione in autonomia e sicurezza del trattamento dialitico, sia esso individuato in dialisi peritoneale domiciliare manuale (CAPD), automatizzata (APD) o in emodialisi domiciliare. Istruisce al corretto riconoscimento e alla gestione delle eventuali complicanze tecnico/infettive connesse al trattamento stesso e alla corretta gestione dell'accesso vascolare (FAV e CVC) e/o del catetere peritoneale;

- è un esperto clinico nell'assistenza nefrologica e contribuisce positivamente alla soddisfazione dei bisogni della comunità;

- è un professionista esperto, il cui scopo principale è far star bene il paziente con le cure appropriate, nel rispetto dei valori culturali, del credo e della dignità della persona.

\section{$7^{\text {a }}$ fase \\ Trapianto di rene}

L'Infermiere agisce nei diversi momenti dall'identificazione dei candidati e dall'inserimento in lista al follow-up, senza dimenticare il coordinamento organizzativo della fase del trapianto.

- Conosce il percorso di trapiantologia e le Linee Guida del Centro Nazionale Trapianti (CNT) e i protocolli dei Centri Trapianto di riferimento, relativi all'inserimento in lista per trapianto da cadavere e da vivente, accompagnando il paziente nelle varie fasi.

- Conosce e utilizza le tecniche di comunicazione e di educazione terapeutica e di gestione delle emozioni correlate al trapianto da vivente e da cadavere.

- È capace di accogliere il paziente nel post-trapianto e di seguire le direttive del Centro Trapianto di riferimento.

\section{Aspetti trasversali}

L'aspetto educativo e relazionale è presente in tutte le aree citate sopra e coinvolge l'Infermiere nell'erogazione di un'assistenza competente e personalizzata, per i pazienti e per le loro famiglie. L'Infermiere provvede in particolare a supportare, educare e prevenire le possibili complicanze, incoraggiando i pazienti verso l'autonomia e l'autocura. È responsabile dei percorsi specifici per il raggiungimento, da parte dell'assistito, di un livello di conoscenze e competenze che gli permetta di gestire bisogni specifici per evitare le possibili complicanze e/o per individuarle e segnalarle il più precocemente possibile. Esplicita un contratto educativo con il paziente e la famiglia, allo scopo di impegnarsi reciprocamente al raggiungimento di obiettivi comuni:

- compliance nei confronti della dieta e dell'assunzione di liquidi; 
- gestione della terapia farmacologica;

- gestione dell'accesso vascolare o peritoneale;

- gestione della metodica dialitica domiciliare (Emodialisi e Dialisi peritoneale);

- controllo del follow-up post-trapianto e gestione del rene trapiantato;

- gestione della relazione di aiuto con la persona assistita e la sua famiglia;

- tutoraggio telefonico nel trattamento dialitico domiciliare per la risoluzione delle problematiche che non richiedono una valutazione ospedaliera;

- individuazione, informazione ed educazione, in collaborazione con l'equipe, dell'eventuale donatore di rene all'interno del nucleo familiare;

- uso e creazione del materiale informativo e visivo specifico, da visionare con il paziente e la famiglia riguardo alle metodiche dialitiche e al trapianto.

\section{Conclusione}

La funzione infermieristica, dettagliata nelle fasi precedentemente descritte e completata dalla Job Description contenuta nel documento completo (I quaderni della formazione OPI, Bologna) (3), trova una sua reale collocazione in tutto il percorso della malattia renale, dalla prevenzione primaria fino al trapianto.

Il codice deontologico infermieristico, revisionato dalla FNOPI (2019), ha chiarito in vari articoli che le competenze degli Infermieri sono anche di natura trasversale, come, per esempio, I'articolo 4, Relazione di cura: “Nell'agire professionale, I'Infermiere stabilisce una relazione di cura, utilizzando l'ascolto e il dialogo. Si fa garante che la persona assistita non sia mai lasciata in abbandono, coinvolgendo, con il consenso dell'interessato, le sue figure di riferimento, nonché le altre figure professionali e istituzionali. Il tempo di relazione è tempo di cura".

L'Infermiere Nefrologico è, quindi, un professionista della salute con adeguate conoscenze nell'assistenza a persone affette da insufficienza renale ad ogni stadio della progressione terapeutica (EDTNA/ERCA Nephrology Nurse Profile, 1994) $(4,5)$ e ha la responsabilità di garantire al singolo e alla collettività l'assistenza infermieristica in considerazione dei bisogni complessivi della persona dalla prevenzione (nel nostro caso della malattia renale) per poi proseguire con la presa in carico, con il follow-up e con la gestione delle complicanze, agendo con una competenza tecnica, relazionale, educativa e organizzativa.

Gli Infermieri continuano ad affrontare molte sfide. La carenza di professionisti a livello globale e la contemporanea riduzione della spesa per i servizi sanitari o la prevenzione delle malattie ha portato a richieste contrastanti sia per i pazienti che per gli operatori sanitari. Nonostante ciò e all'interno di questi vincoli, gli Infermieri di nefrologia e i loro colleghi hanno continuato a guidare cambiamenti significativi e miglioramenti ai servizi e agli standard, per fornire la massima qualità dell'assistenza ai pazienti con malattia renale (4).

\section{Acknowledgements}

Gli autori ringraziano tutti i componenti del gruppo di lavoro per la collaborazione prestata nello svolgimento di questo studio:

- Ballarini Melissa (AUSL di Bologna)

- Bellini Antonella (Nefrologia Az. Osp. S. Orsola Malpighi)

- Bonori Veronica (Infermiere Dialisi Az. Osp. S. Orsola Malpighi)

- Brintazzoli Silvia (Infermiere Dialisi AUSL di Bologna)

- Elia Cinzia (Infermiere Dialisi Az. Osp. S. Orsola Malpighi)

- Fabbri Cinzia (Coordinatore Inf.co Dialisi peritoneale Az. Osp. S. Orsola Malpighi - Presidente SIAN)

- Giurdanella Pietro (Coordinatore Inf.co Nefrologia e Dialisi Az. Osp. S. Orsola Malpighi e Presidente Ordine delle Professioni Infermieristiche di Bologna)

- Longo Walter (Infermiere Nefrologia Az. Osp. S. Orsola Malpighi)

- Oliva Domenico (Dipartimento Cure Primarie AUSL di Bologna)

- Presentati Francesca (Infermiere Dialisi Az. Osp. S. Orsola Malpighi)

- Russo Maria (Infermiere Dialisi AUSL di Bologna)

- Toschi Roberta (Responsabile Processi Assistenziali Dialitici AUSL di Bologna e Consigliere Ordine delle Professioni Infermieristiche di Bologna)

- Zito Maria Pia (Infermiere Case Manager Dialisi Peritoneale Az. Osp. S. Orsola Malpighi sino 2018 e Consiglio Direttivo SIAN)

\section{Disclosures}

Conflict of interest: The authors declare no conflict of interest. Financial support: This research received no specific grant from any funding agency in the public, commercial, or not-for-profit sectors.

\section{Bibliografia}

1. Pegoraro M, Zito MP, Galeotti P, Delalio A, Rossi F, Guadagno V. Infermieristica Nefrologica: Competenze Assistenziali Generali e Specifiche. G Tec Nefrol Dial. 2014;26(1):42-49. CrossRef

2. Documento di Indirizzo per la Malattia Renale Cronica. 2014 Online (data di accesso Luglio 2021).

3. Quaderni della Formazione OPI Bologna 2021 - "Presa in carico infermieristica nel percorso della Malattia Renale Cronica". Online (data di accesso luglio 2021).

4. Perché l'analisi delle competenze L'infermiere. 2007;4:2-40 Online (data di accesso Luglio 2021).

5. The Profile of Nephrology Nursing. 2018. European Dialysis and Transplant Nurses Association/European Renal Care Association (EDTNA/ERCA). Online (data di accesso Luglio 2021). 